\title{
Existence of cooperative search technique to find a Brownian target
}

\author{
Mohamed Abd Allah El-Hadidy ${ }^{1,2}$
}

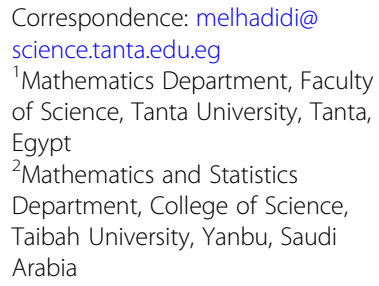

Correspondence: melhadidi@ science.tanta.edu.eg

${ }^{1}$ Mathematics Department, Faculty of Science, Tanta University, Tanta, Egypt

${ }^{2}$ Mathematics and Statistics Department, College of Science, Taibah University, Yanbu, Saudi Arabia

\begin{abstract}
This article aims to study the existence of the cooperative search technique to find a Brownian target. We have $2 n$ cooperative searchers coordinate their search to find a Brownian target that moves on one of $n$ disjoint real lines. Each line has two searchers. All of these searchers start the searching process from the origin. Rather than finding the conditions that make the expected value of the first interviewing time between one of the searchers and the target is finite, we compute the approximate value of this expected value.
\end{abstract}

Keywords: Search theory, Coordinated search technique, Probability theory, Brownian motion

Mathematics subject classification: 37A50, 60 K30

\section{Introduction}

The searching problem for the lost targets that move with random movement is one of the most important contemporary problems that the mathematicians seek to solve it in different ways to reduce the cost of the search. One of these random movements is the Brownian motion. In an earlier work, this problem has been discussed on the real line, plan, and space by studying different kinds of search plans such as the linear search plan as in El-Hadidy et al [1, 2]., El-Rayes et al. [3], and Corwin [4]. They presented the finiteness of the search strategy which was used by founding the conditions that make $E(\tau)$ (the first interviewing time expected value for one of the searchers and the target where $\tau$ denote to this time) finite. In addition, they showed the existence of this optimal search plan and El-Hadidy [1] found it.

One of the famous search techniques is the coordinated search technique. Reyniers $[5,6]$ was the first one who investigated this technique on the line. This technique considered two searchers starting together at the origin of the real line with unit speed. These searchers aimed to find the expected time for detecting a hidden target after one of them return to the origin to tell the other. In addition, they aimed to minimize this expected time. But, the returning time increases this expected time. Here, we will treat this defect by not repeating the searching process on the searched parts.

In recent works, El-Hadidy and Abou-Gabal [7] presented a coordinated search technique to find a random Walk target, which moves randomly on the real line. Rather than showing the existence of the optimal search strategy that minimizes this first interviewing time, El-Hadidy and Abou-Gabal [7] found the conditions that make $E(\tau)$

(c) The Author(s). 2020 Open Access This article is distributed under the terms of the Creative Commons Attribution 4.0 International License (http://creativecommons.org/licenses/by/4.0/), which permits unrestricted use, distribution, and reproduction in any medium, provided you give appropriate credit to the original author(s) and the source, provide a link to the Creative Commons license, and indicate if changes were made. 
finite. El-Hadidy et al. [8-10] also studied this technique on the plan and space when the target is hidden at any random point. They found the expected value of the time to detect the target by one of the searchers. Besides that, they found the optimal search plan that minimizes this expected value. For different kinds of search plans on the line, plan, and space, the reader can see, El-Hadidy et al. [11-20], Kagan and Ben-Gal [21], Guerrier and Holcman [22], Palyulin et al. [23], Radmard and Croft [24], Stone et al. [25], and Jia et al. [26].

In this work, we use the technique which was presented in Reyniers $[5,6]$ and ElHadidy and Abou-Gabal [7] but without repeating the searching process on the searched parts of $n$ disjoint real lines. This technique is the generalization of the technique which was used in El-Hadidy and Alzulaibani [27]. We use $n$ cooperative searchers to seek a Brownian target which moves in one of the $n$ disjoint cylinders (real lines). This idea is supported through modern communication methods. Each line has two searchers starting at the same time from the origin. One of these searchers goes towards $+\infty$ to search the right part of the real line and the other goes towards to $-\infty$ to search the left part of the real line. This will reduce $E(\tau)$ (cost). Thus, we aim to show the existence of a finite search plan and compute this expected value.

The organization of this paper appears as follows: the "Problem formulation" section describes our search problem. We show the existence and finiteness of our search plan in the "Existence of the finite search plan" section. The computation of $E(\tau)$ appears in "Computational expected value for $\tau$ " section. Finally, we discuss the results and future works which were obtained in the conclusion part.

\section{Problem formulation}

We always seek for reducing the cost to detect the Brownian moving target on one of the $n$ real lines. To do this, we use $n$ cooperative searchers as in El-Hadidy et al. [1, 2, 20, 28]. But, the technique which was used in El-Hadidy et al. [1, 2, 20, 28] wasted a lot of costs because the searching process is repeated in the same part more than once. Our model does not waste this cost because the searchers do not repeat the searching process by using the connection technology methods. Besides that, we calculate the approximated expected value of the Brownian target detection.

The searching framework

The space of search

The search space is the $n$ axis of $n$ cylinders ( $n$ real lines $L_{i}, i=1,2, \ldots, n$ ), see Fig. 1 .

\section{The target}

Our target moves with a Brownian motion $\{B(t), t \geq 0\}$, where the target's initial position is unknown, but its probability distribution is known at time 0 .

\section{The means of search}

All the searchers $S_{j}, j=1,2, \ldots, 2 n$ start the searching process from the origin. Each line $L_{i}, i=1,2, \ldots, n$ needs two unit speed searchers $S_{2 j-1}$ and $S_{2 j}$, where the searcher $S_{2 j-1}$ searches in the right part and $S_{2 j}$ in the left part of the real line. After searching successively common distances, they do not return to the origin of $L_{i}$. The searching process 


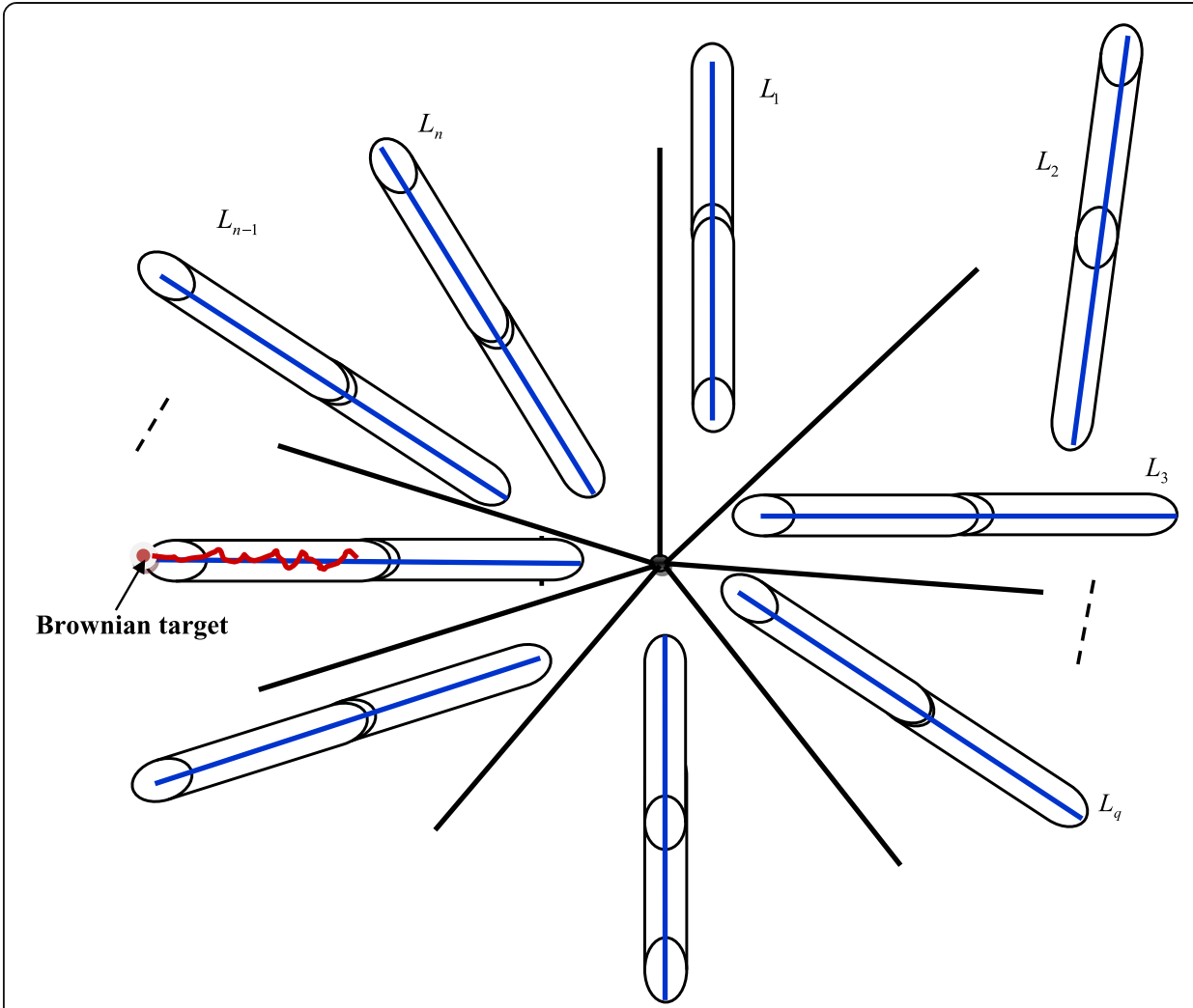

Fig. 1 The search space ( $n$ disjoint cylinders)

on $L_{i}$ would conduct as follows: begin at $H_{0 i}=0$ and go to the right (left) as far as $H_{1 i}^{(+)}$ $\left(H_{2 i}^{(-)}\right)$. Then, the first one of $S_{2 j-1}$ and $S_{2 j}$ which meets the target will tell the other by using the wireless communication methods. If the target is not found, then do the above step again at the point $H_{1 i}^{(+)}\left(H_{2 i}^{(-)}\right)$to search the right (left) part of $H_{1 i}^{(+)}\left(H_{2 i}^{(-)}\right)$as far as $\left[H_{3 i}^{(+)}-H_{1 i}^{(+)}\right]\left(\left[\left|H_{4 i}^{(-)}-H_{2 i}^{(-)}\right|\right]\right)$and so on, see Fig. 2.

\section{Existence of the finite search plan}

In this section, we aim to get the conditions that make the search plan be finite. On the line $L_{i}, i=1,2, \ldots, n$ the two searchers $S_{2 j-1}$ and $S_{2 j}$ are coordinating their search

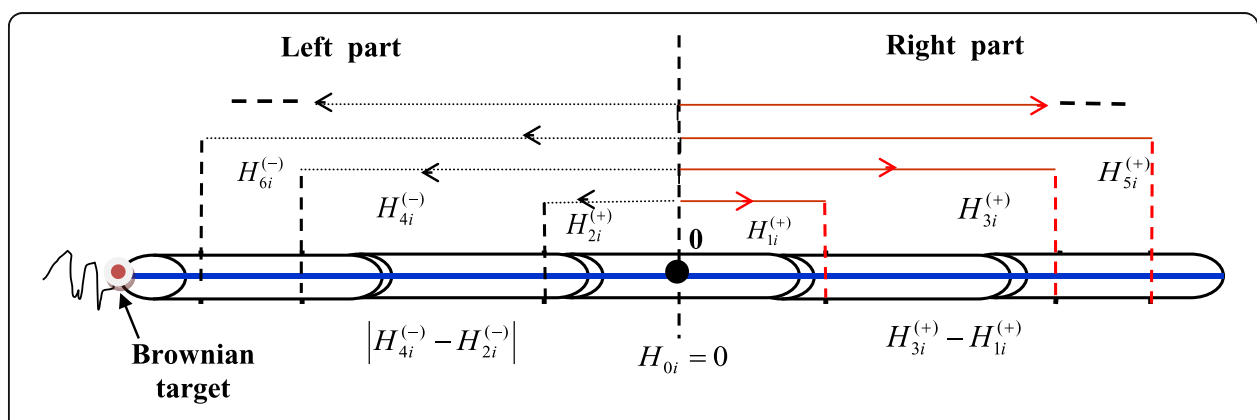

Fig 2 The search plan without returning to the origin of $S_{2 j-1}$ and $S_{2 j}$ for finding a Brownian target on $L_{i}$ 
and following the search paths $\phi_{2 j-1}: R^{+} \rightarrow R$ and $\bar{\phi}_{2 j}: R^{+} \rightarrow R$, respectively, to meet the target, where

$$
\left|\phi_{2 j-1}\left(t_{1}\right)-\phi_{2 j-1}\left(t_{2}\right)\right| \leq v^{(+)}\left|t_{1}-t_{2}\right| \&\left|\bar{\phi}_{2 j}\left(t_{1}\right)-\bar{\phi}_{2 j}\left(t_{2}\right)\right| \leq v^{(-)}\left|t_{1}-t_{2}\right| \forall t_{1}, t_{2} \in R^{+},
$$

(i.e., the search paths should satisfy the Lipschitz condition), and $v^{(+)}$and $v^{(-)}$are the velocities of the searchers in the right and left part, respectively. Assuming that $\theta_{i(+)}$, $\theta_{i(-)}, \lambda_{i(+)}$, and $\lambda_{i(-)}$ are positive integer numbers greater than one. For any line, $L_{i}, i=1$, $2, \ldots, n$ define the sequences $\left\{G_{k i}^{(+)}\right\}_{k \geq 1, i=1,2, \ldots, n},\left\{G_{k i}^{(-)}\right\}_{k \geq 1, i=1,2, \ldots, n},\left\{H_{k i}^{(+)}\right\}_{k \geq 1, i=1,2, \ldots, n}$ and $\left\{H_{k i}^{(-)}\right\}_{k \geq 1, i=1,2, \ldots, n}$ (see El-Rayes et al. [3]), respectively, to obtain the distances

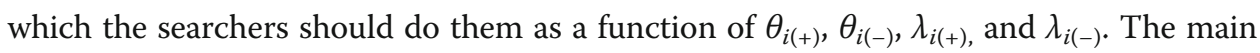
idea of this model is all searchers do not return to the origin as in Fig. 1 that is reducing $E(\tau)$. Thus, we can define $G_{i k}^{(+)}=\lambda_{i(+)}\left(\theta_{i(+)}^{k}-1\right), G_{i k}^{(-)}=\lambda_{i(-)}\left(\theta_{i(-)}^{k}-1\right)$. This leads to the traveled distances at the time step $k, k=1,2, \ldots$ are given by $H_{k i}^{(+)}=C_{i}^{(+)}\left(G_{k i}^{(+)}+2\right)$ ,$H_{k i}^{(-)}=C_{i}^{(-)}\left(G_{k i}^{(-)}+2\right)$, in the right and left part of $L_{i}, i=1,2, \ldots, n$, respectively, where $C_{i}^{(+)}=\frac{\varpi_{i}^{(+)}\left(\theta_{i(+)}-1\right)}{\left(\theta_{i(+)}+1\right)}, C_{i}^{(-)}=\frac{\varpi_{i}^{(-)}\left(\theta_{i(-)}-1\right)}{\left(\theta_{i(-)}+1\right)}$, and $\varpi_{i}^{(+)}, \varpi_{i}^{(-)}$are rational numbers. Consequently, the search path in the right part of $L_{i}, i=1,2, \ldots, n$ can be defined as follows: for any $t \in R^{+}$, if $G_{(2 k-3) i}^{(+)} \leq t \leq G_{(2 k-1) i}^{(+)}$, we have

$$
\phi_{i}(t)=\left(H_{(2 k-1) i}^{(+)}-H_{(2 k-3) i}^{(+)}\right)+\left(t-G_{(2 k-1) i}^{(+)}\right)
$$

And, in the left part for any $t \in R^{+}$, if $G_{(2 k-2) i}^{(-)} \leq t \leq G_{(2 k) i}^{(-)}$, we have

$$
\bar{\phi}_{i}(t)=-\left[\left(H_{(2 k) i}^{(-)}-H_{(2 k-2) i}^{(-)}\right)+\left(t-G_{(2 k) i}^{(-)}\right)\right]
$$

Let the notations $\psi\left(G_{(2 k-1) i}^{(+)}\right)=B\left(G_{(2 k-1) i}^{(+)}\right)-C_{i}^{(+)}\left(G_{(2 k-1) i}^{(+)}\right), \tilde{\psi}\left(G_{(2 k) i}^{(-)}\right)=B\left(G_{(2 k) i}^{(-)}\right)$ $+C_{i}^{(-)}\left(G_{(2 k) i}^{(-)}\right), i=1,2, \ldots, n ; k=1,2, \ldots$ are held. Then,

$$
\left.\tau=\inf \left\{t: \text { either one of } \phi_{i}(t)=Z_{0 i}+B(t) \text { or } \bar{\phi}_{i}(t)=Z_{0 i}+B(t)\right\}, i=1,2, \ldots, n\right\},
$$

where $Z_{0 i}$ is a random variable which represents the target's initial position on $L_{i}, i=1,2$, ..., $n$ and independent with $\{B(t), t>0\}$. Also, let the search plan be represented by $\left(\phi_{1}, \phi_{2}\right.$, $\left.\ldots, \phi_{n}, \bar{\phi}_{1}, \bar{\phi}_{2}, \ldots, \bar{\phi}_{n}\right) \in \Phi_{0}$, where $\Phi_{0}=\left\{(\Phi, \bar{\Phi}): \phi_{i} \in \Phi, \bar{\phi}_{i} \in \bar{\Phi} \forall i=1,2, \ldots, n\right\}$.

For $t>0$, we have an infinite number of possible outcomes, thus the probability density function which represents the target's position can be described as a calendar for the continuity of the relative frequencies of the data in a given interval. Consequently, the probability of any certain value of the target's position at time step $k$ is equal to zero. Logically, the first interviewing time event and the target's position $z_{i} \in Z_{i}$ on any line $L_{i}, i=1,2, \ldots, n$ at time step $k$ on $[0, \infty]$ or $[-\infty, 0]$ have a known probability value greater than zero. This leads us to study our problem in a new space (probability space). Let this probability space be $(\Omega, \Sigma, \gamma)$ where $\Omega$ is the sample space of all expected meeting points, $\Sigma$ is the $\sigma$ - algebra which represents the collection of all mutually exclusive events that show the target's position at any time step $k$, and $\gamma$ is the probability measure which used as a measure of an integrator factor into our 
probability space. Now, we will study the existence of the finite search plan as in the following theorems.

Theorem 1: The combination of the search plans $\left(\phi_{1}, \phi_{2}, \ldots, \phi_{n}, \bar{\phi}_{1}, \bar{\phi}_{2}, \ldots, \bar{\phi}_{n}\right) \in \Phi_{0}$ is finite if the following $\int_{0}^{\infty} \sum_{k=2}^{\infty} \theta_{i(+)}^{2 k-2} P\left(\psi\left(G_{(2 k-3) i}^{(+)}\right)>-z_{i}\right) \gamma_{i}\left(d z_{i}\right)$ and $\int_{-\infty}^{0} \sum_{k=2}^{\infty} \theta_{(-)}^{2 i-2} P\left(\tilde{\psi}\left(G_{(2 k-2)}^{(-)}\right)\right.$ $\left.<-z_{i}\right) \gamma_{i}\left(d z_{i}\right)$ are finite for all $i=1,2, \ldots, n$, where $\sum_{i=1}^{n} \gamma_{i}=1$.

Proof Since the target may be met at one of $n$ lines, then $\tau_{\phi_{i}}, i=1,2, \ldots, n$ "the first interviewing time between one of the searchers $S_{2 j-1}, j=1,2, \ldots, 2 n$ in the right parts of all lines $L_{i}, i=1,2, \ldots, n$ and the Brownian target" and $\tau_{\bar{\phi}_{i}}, i=1,2, \ldots, n$ "the first interviewing time between one of the searchers $S_{2 j}, j=1,2, \ldots, 2 n$ in the left parts and the Brownian target" of all lines $L_{i}, i=1,2, \ldots, n$ are mutually exhaustive events. Then, for any $k \geq 0$, we have

$$
\begin{gathered}
P(\tau>t)=P\left(\tau_{\phi_{1}}>t \text { or } \tau_{\phi_{2}}>t \text { or } \ldots \text { or } \tau_{\phi_{n}}>t \text { or } \tau_{\bar{\phi}_{1}}>t \text { or } \tau_{\bar{\phi}_{2}}>t \text { or } \ldots \text { or } \tau_{\bar{\phi}_{n}}>t\right) \\
=\underbrace{n}_{i=1}\left(P\left(\tau_{\phi_{i}}>t\right)+P\left(\tau_{\bar{\phi}_{i}}>t\right)\right) \\
=\sum_{i=1}^{n}\left(P\left(\tau_{\phi_{i}}>t\right)+P\left(\tau_{\bar{\phi}_{i}}>t\right)\right) .
\end{gathered}
$$

Then, we obtain

$$
\begin{gathered}
E(\tau)=\int_{0}^{\infty} P(\tau>t) d t \leq \sum_{1=1}^{n} \sum_{k=1}^{\infty}\left[\int_{G_{(2 k-3) i}^{+(-3)}}^{G_{(2 k-1) i}^{(+)}} P\left(\tau_{\phi_{i}}>t\right) d t+\int_{G_{(2 k-2) i}^{(-)}} P\left(\tau_{\bar{\phi}_{i}}>t\right) d t\right] \\
\left.\leq \sum_{i=1}^{n} \sum_{k=1}^{\infty}\left[\left(G_{(2 k-1) i}^{(+)}-G_{(2 k-3) i}^{(+)}\right) P\left(\tau_{\phi_{i}}>G_{(2 k-3) i}^{(+)}\right)\right)+\left(G_{(2 k) i}^{(-)}-G_{(2 k-2) i}^{(-)}\right) P\left(\tau_{\bar{\phi}_{i}}>G_{(2 k-2) i}^{(-)}\right)\right] \\
\leq \sum_{i=1}^{n} \sum_{k=1}^{\infty}\left[\lambda_{i(+)}\left(\theta_{i(+)}^{2 k-1}-\theta_{i(+)}^{2 k-3}\right) P\left(\tau_{\phi_{i}}>G_{(2 k-3) i}^{(+)}\right)+\lambda_{i(-)}\left(\theta_{i(-)}^{2 k}-\theta_{i(-)}^{2 k-2}\right) P\left(\tau_{\bar{\phi}_{i}}>G_{(2 k-2) i}^{(-)}\right)\right] \\
=\sum_{i=1}^{n}\left(\lambda_{i(+)} \theta_{i(+)} P\left(\tau_{\phi_{i}}>0\right)+\lambda_{i(+)} \theta_{i(+)}\left(\theta_{i(+)}^{2}-1\right) \sum_{k=2}^{\infty} \theta_{i(+)}^{2 k-2} P\left(\tau_{\phi_{i}}>G_{(2 k-3) i}^{(+)}\right)\right. \\
\left.+\lambda_{i(-)} \theta_{i(-)} P\left(\tau_{\overline{\phi_{i}}}>0\right)+\lambda_{i(-)} \theta_{i(-)}^{2}\left(\theta_{i(-)}^{2}-1\right) \sum_{k=2}^{\infty} \theta_{(-)}^{2 k-2} P\left(\tau_{\overline{\phi_{i}}}>G_{(2 k-2) i}^{(-)}\right)\right) .
\end{gathered}
$$

Also, at time step $k$ on the line $L_{i}, i=1,2, \ldots, n$, we have

$$
P\left(\tau_{\phi_{i}}>G_{(2 k-3) i}^{(+)}\right) \leq \int_{0}^{\infty} P\left(Z_{0 i}+B\left(G_{(2 k-3) i}^{(+)}\right)>H_{(2 k-3) i}^{(+)} \mid Z_{0 i}=z_{i}\right) \gamma_{i}\left(d z_{i}\right), k \geq 1 .
$$

By using the above notation $\psi\left(G_{(2 k-3) i}^{(+)}\right)=B\left(G_{(2 k-3) i}^{(+)}\right)-C_{i}^{(+)}\left(G_{(2 k-3) i}^{(+)}\right)$, we can get $\psi\left(G_{(2 k-3) i}^{(+)}\right)>-z_{i}$ (El-Hadidy et al. [1, 2]), then $P\left(\tau_{\phi_{i}}>G_{(2 k-3) i}^{(+)}\right) \leq \int_{0}^{\infty} P\left(\psi\left(G_{(2 k-3) i}^{(+)}\right)>-z_{i}\right) \gamma_{i}\left(d z_{i}\right)$, and by using the another notation $\tilde{\psi}\left(G_{(2 k) i}^{(-)}\right)=B\left(G_{(2 k) i}^{(-)}\right)+C_{i}^{(-)}\left(G_{(2 k) i}^{(-)}\right)$, we get $P\left(\tau_{\bar{\phi}_{i}}>G_{(2 k-2)}^{(-)}\right) \leq \int_{-\infty}^{0} P\left(\tilde{\psi}\left(G_{(2 k-2) i}^{(-)}\right)<-z_{i}\right) \gamma_{i}\left(d z_{i}\right)$. Consequently,

$$
E(\tau)=\sum_{i=1}^{n}\left[g_{i}+\lambda_{i(+)} \theta_{i(+)}\left(\theta_{i(+)}^{2}-1\right) \int_{0}^{\infty} M_{i}\left(z_{i}\right) \gamma_{i}\left(d z_{i}\right)+\lambda_{i(-)} \theta_{i(-)}^{2}\left(\theta_{i(-)}^{2}-1\right) \int_{-\infty}^{0} L_{i}\left(z_{i}\right) \gamma_{i}\left(d z_{i}\right)\right],
$$

where $g_{i}=\left(\lambda_{i(+)} \theta_{i(+)}+\lambda_{i(-)} \theta_{i(-)}\right) P(\tau>0), P(\tau>0)$ is the knowing initial probability of $\tau$ and $M_{i}\left(z_{i}\right)=\sum_{k=2}^{\infty} \theta_{i(+)}^{2 k-2} P\left(\psi\left(G_{(2 k-3) i}^{(+)}\right)>-z_{i}\right)$ and $L_{i}\left(z_{i}\right)=\sum_{k=2}^{\infty} \theta_{i(-)}^{2 k-2} P\left(\tilde{\psi}\left(G_{(2 k-2) i}^{(-)}\right)<-z_{i}\right)$. Thus, $E(\tau)$ is finite if $\int_{0}^{\infty} M_{i}\left(z_{i}\right) \gamma_{i}\left(d z_{i}\right)$ and $\int_{-\infty}^{0} L_{i}(z) \gamma_{i}\left(d z_{i}\right)$ are finite. The prove is completed. 
This model is the first investigation of the cooperative search technique by using $2 n$ searchers which reduces $E(\tau)$. But the above conditions in Theorem 1 are not sufficient to get the finite expected search time. Thus, we want to get more conditions to make this model more applicable and effective.

Theorem 2: At any time step $k$, the chosen search plan $\Phi_{0}=\left\{(\Phi, \bar{\Phi}): \phi_{i} \in \Phi, \bar{\phi}_{i} \in \bar{\Phi} \forall i\right.$ $=1,2, \ldots, n\}$ should satisfy $\left[M_{1}\left(z_{k 1}\right), M_{2}\left(z_{k 2}\right), \ldots, M_{n}\left(z_{k n}\right)\right] \leq\left[\tilde{M}_{1}\left(\left|z_{k 1}\right|\right), \tilde{M}_{2}\left(\left|z_{k 2}\right|\right), \ldots, \tilde{M}_{n}\right.$ $\left.\left(\left|z_{k n}\right|\right)\right]$ and $\left[L_{1}\left(z_{k 1}\right), L_{2}\left(z_{k 2}\right), \ldots, L_{n}\left(z_{k n}\right)\right] \leq\left[\tilde{L}_{1}\left(\left|z_{k 1}\right|\right), \tilde{L}_{2}\left(\left|z_{k 2}\right|\right), \ldots, \tilde{L}_{n}\left(\left|z_{k n}\right|\right)\right]$ where $\left[\tilde{M}_{1}\left(\left|z_{k 1}\right|\right.\right.$ )$\left., \tilde{M}_{2}\left(\left|z_{k 2}\right|\right), \ldots, \tilde{M}_{n}\left(\left|z_{k n}\right|\right)\right]$ and $\left[\tilde{L}_{1}\left(\left|z_{k 1}\right|\right), \tilde{L}_{2}\left(\left|z_{k 2}\right|\right), \ldots, \tilde{L}_{n}\left(\left|z_{k n}\right|\right)\right]$ are vectors of linear functions.

Proof At time step $k$, if $z_{k i} \leq 0$ on the line $L_{i}, i=1,2, \ldots, n$, then $\left[M_{1}\left(z_{k 1}\right), M_{2}\left(z_{k 2}\right), \ldots\right.$, $\left.M_{n}\left(z_{k n}\right)\right] \leq\left[M_{1}(0), M_{2}(0), \ldots, M_{n}(0)\right]$ but for $z_{k i}>0$, we get $M_{i}(0)=\sum_{k=2}^{\infty} \theta_{i(+)}^{2 k-2} P\left(\psi\left(G_{(2 k-1) i}^{(+)}\right)\right.$ $>0)$. Consequently,

$$
\begin{aligned}
{\left[M_{1}\left(z_{k 1}\right), M_{2}\left(z_{k 2}\right), \ldots, M_{n}\left(z_{k n}\right)\right]=\left[M_{1}(0)\right.} & +\sum_{k=2}^{\infty} \theta_{1(+)}^{2 k-2} P\left(\psi\left(G_{(2 k-1) 1}^{(+)}\right) \leq 0\right), M_{2}(0)+\sum_{k=2}^{\infty} \theta_{2(+)}^{2 k-2} P\left(\psi\left(G_{(2 k-1) 2}^{(+)}\right) \leq 0\right), \ldots, M_{n}(0) \\
& \left.+\sum_{k=2}^{\infty} \theta_{n(+)}^{2 k-2} P\left(\psi\left(G_{(2 k-1) n}^{(+)}\right) \leq 0\right)\right] .
\end{aligned}
$$

If the target starts its motion at time step $k$ on the real line $L_{i}, i=1,2, \ldots, n$ from the random point $z_{k i}$ with drift $\mu$ and variance $\sigma^{2}$, then on the right part of $L_{i}$ and for $t \geq$ $G_{(2 k-1) i}^{(+)}>0$, we have

$$
P\left(B(t) \geq \alpha_{i} t\right) \leq P\left(B\left(G_{(2 k-1) i}^{(+)}\right) \geq \alpha_{i} G_{(2 k-1) i}^{(+)}\right)=P\left(\sigma \sqrt{G_{(2 k-1) i}^{(+)}} Z+\mu G_{(2 k-1) i}^{(+)} \geq \alpha_{i} G_{(2 k-1) i}^{(+)}\right),
$$

where $\alpha_{i}, i=1,2, \ldots, n$ are constants. This leads to

$$
p\left(Z \geq \frac{\left(\alpha_{i}-\mu\right) G_{(2 k-1) i}^{(+)}}{\sigma \sqrt{G_{(2 k-1) i}^{(+)}}}\right)=\int_{k}^{\infty} \frac{1}{\sqrt{2 \pi}} e^{-\frac{z^{2}}{2}} d z=\int_{0}^{\infty} \frac{1}{\sqrt{2 \pi}} e^{-\frac{\left(x+\kappa_{i}\right)^{2}}{2}} d x \leq \frac{1}{2} \varepsilon^{G_{(2 k-1) i}^{(+)}},
$$

where $\kappa_{i}=\frac{\left(\alpha_{i}-\mu\right) G_{(2 k-1) i}^{(+)}}{\sigma \sqrt{G_{(2 k-1) i}^{(+)}}}, i=1,2, \ldots, n$. Also, in the left part of $L_{i}$, we can get

$$
P\left(Z<\frac{\left(\alpha_{i}-\mu\right) G_{(2 k-2) i}^{(-)}}{\sigma \sqrt{G_{(2 k-2) i}^{(-)}}}\right) \leq 1-\frac{1}{2} \varepsilon^{G_{(2 k-2) i}^{(-)}} .
$$

From (7) and (8) in (6), we obtain

$\left[M_{1}(0), M_{2}(0), \ldots, M_{n}(0)\right]<\left[\sum_{k=2}^{\infty} \theta_{1(+)}^{2 k-2} \varepsilon_{1}^{G_{(2 k-1) 1}^{(+)}}, \sum_{k=2}^{\infty} \theta_{2(+)}^{2 k-2} \varepsilon_{2}^{G_{(2 k-1) 2}^{(+)}}, \ldots, \sum_{k=2}^{\infty} \theta_{n(+)}^{2 k-2} \varepsilon_{n}^{G_{(2 k-1) n}^{(+)}}\right], \quad 0<\varepsilon_{i}<1, i=1,2, \ldots, n$.

For any two random positions $z_{2 i} \leq z_{1 i}$ of the target on the right part of $L_{i}$, we get $P\left(z_{2 i} \leq B(t) \leq z_{1 i}\right) \leq P\left(z_{2 i} \leq B\left(G_{(2 k-1) i}^{(+)}\right) \leq z_{1 i}\right)$ is non-increasing with time $G_{(2 k-1) i}^{(+)}$, where $t \geq G_{(2 k-1) i}^{(+)} \geq \max \left(\frac{z_{1 i}}{\mu}, \frac{z_{2 i}}{\mu}\right)$,

because 


$$
\begin{gathered}
\left.P\left(z_{2 i} \leq B\left(G_{(2 k-1) i}^{(+)}\right) \leq z_{1 i}\right)=P\left(z_{2 i} \leq \sigma \sqrt{G_{(2 k-1) i}^{(+)}}\right) Z+\mu G_{(2 k-1) i}^{(+)} \leq z_{1 i}\right) \\
=P\left(\frac{z_{2 i}-\mu G_{(2 k-1) i}^{(+)}}{\sigma \sqrt{G_{(2 k-1) i}^{(+)}}} \leq Z \leq \frac{z_{1 i}-\mu G_{(2 k-1) i}^{(+)}}{\sigma \sqrt{G_{(2 k-1) i}^{(+)}}}\right) .
\end{gathered}
$$

If $\mu<0$, then $\frac{z_{2 i}-\mu G_{(2 k-1) i}^{(+)}}{\sigma \sqrt{G_{(2 k-1) i}^{(+)}}} \geq 0, \frac{d}{d G_{(2 k-1) i}^{(+)}}\left(\frac{z_{2 i}-\mu G_{(2 k-1) i}^{(+)}}{\sigma \sqrt{G_{(2 k-1) i}^{(+)}}}\right) \geq 0, \frac{z_{1 i}-\mu G_{(2 k-1) i}^{(+)}}{\sigma \sqrt{G_{(2 k-1) i}^{(+)}}} \leq 0$ and $\frac{d}{d G_{(2 k-1) i}^{(+)}}\left(\frac{z_{1 i}-\mu G_{(2 k-1) i}^{(+)}}{\sigma \sqrt{G_{(2 k-1) i}^{(+)}}}\right) \leq 0$.

Thus, $P\left(z_{2 i} \leq B\left(G_{(2 k-1) i}^{(+)}\right) \leq z_{1 i}\right)$ be non-increasing.

Assuming that $\left[\psi_{1}(k), \psi_{2}(k), \ldots, \psi_{n}(k)\right]=\left[\sum_{\ell=1}^{k} y_{\ell 1}, \sum_{\ell=1}^{k} y_{\ell 2}, \ldots, \sum_{\ell=1}^{k} y_{\ell n}\right]$, where $\left\{y_{\ell i}\right\}_{\ell \geq 1, i=1}$, $2, \ldots, n$ is a sequence of i.i.d.r.vs which represents the target position at time step $k$ on the line $L_{i}, i=1,2, \ldots, n$ and $y_{i k} \sim N\left(\mu-\alpha_{i}, \sigma^{2}\right)$. Consider the following: $\left[d_{k 1}, d_{k 2}, \ldots, d_{k n}\right]$ $=\left[G_{(2 k+1) 1}^{(+)}, G_{(2 k+1) 2}^{(+)}, \ldots, G_{(2 k+1) n}^{(+)}\right]=\left[\lambda_{1(+)}\left(\theta_{1(+)}^{2 k+1}-1\right), \lambda_{2(+)}\left(\theta_{2(+)}^{2 k+1}-1\right), \ldots, \lambda_{n(+)}\left(\theta_{n(+)}^{2 k+1}-1\right)\right]$ and $\quad\left[U_{1}(j, j+1), U_{2}(j, j+1), \ldots, U_{n}(j, j+1)\right]=\left[\sum_{k=1}^{\infty} P\left[-(j+1)<\psi_{1}(k) \leq-j\right], \sum_{k=1}^{\infty} P[-(j\right.$ $\left.\left.+1)<\psi_{2}(k) \leq-j\right], \ldots, \sum_{k=1}^{\infty} P\left[-(j+1)<\psi_{n}(k) \leq-j\right]\right]$. By choosing $\left[d_{m 1}, d_{m 2}, \ldots, d_{m n}\right]=[$ $\left.\max \left(0, \frac{z_{m 1}}{\mu}\right), \max \left(0, \frac{z_{m 2}}{\mu}\right), \ldots, \max \left(0, \frac{z_{m n}}{\mu}\right)\right]$ and also for any line $L_{i}, i=1,2, \ldots, n$, we choose $a_{i}(k)=P\left[-z_{k i}<\psi_{i}(k) \leq 0\right]=\sum_{j=1}^{\left|z_{k i}\right|} P\left[-(j+1)<\psi_{i}(n) \leq-j\right]$. Thus, if $k>d_{m i}$ for all $k \geq 1$, $i=1,2, \ldots, n$ and since $P\left(z_{2 i} \leq B\left(G_{(2 k-1) i}^{(+)}\right) \leq z_{1 i}\right)$ is non-increasing with time $G_{(2 k-1) i}^{(+)}$at step $k$, then we have $a_{i}(k)$ is a non-increasing also. Consequently, $\left[M_{1}\left(z_{k 1}\right), M_{2}\left(z_{k 2}\right), \ldots\right.$, $\left.M_{n}\left(z_{k n}\right)\right]-\left[M_{1}(0), M_{2}(0), \ldots, M_{n}(0)\right]=\left[\sum_{k=1}^{m} \theta_{1(+)}^{2 k+1} a_{1}\left(d_{k 1}\right)+\alpha_{1} \sum_{k=m+1}^{\infty}\left(d_{k 1}-d_{(k-1) 1}\right) a_{1}\left(d_{k 1}\right), \sum_{k=1}^{m}\right.$ $\left.\theta_{2(+)}^{2 k+1} a_{2}\left(d_{k 2}\right)+\alpha_{2} \sum_{k=m+1}^{\infty}\left(d_{k 2}-d_{(k-1) 2}\right) a_{2}\left(d_{k 2}\right), \ldots, \sum_{k=1}^{m} \theta_{n(+)}^{2 k+1} a_{n}\left(d_{k n}\right)+\alpha_{n} \sum_{k=m+1}^{\infty}\left(d_{k n}-d_{(k-1) n}\right) a_{n}\left(d_{k n}\right)\right]$.

From Lemma 1 in El-Hadidy et al [1], we have $\left[M_{1}\left(z_{k 1}\right), M_{2}\left(z_{k 2}\right), \ldots, M_{n}\left(z_{k n}\right)\right]-\left[M_{1}(0)\right.$, $\left.M_{2}(0), \ldots, M_{n}(0)\right] \leq\left[\sum_{k=1}^{m} \theta_{1(+)}^{2 k+1}+\alpha_{1} \sum_{k=d_{m}}^{\infty} a_{1}(k), \sum_{k=1}^{m} \theta_{2(+)}^{2 k+1}+\alpha_{2} \sum_{k=d_{m}}^{\infty} a_{2}(k), \ldots \sum_{k=1}^{m} \theta_{n(+)}^{2 k+1}+\alpha_{2} \sum_{k=d_{m}}^{\infty} a_{n}\right.$
$(k)] \leq\left[d_{m 1}+\lambda_{1(+)}\left(1-\theta_{n(+)}\right) \sum_{j=0}^{\left|z_{k 1}\right|} U_{1}(j, j+1), d_{m 2}+\lambda_{2(+)}\left(1-\theta_{2(+)}\right)+\sum_{j=0}^{\left|z_{k 2}\right|} U_{2}(j, j+1), \ldots d_{m n}\right.$
$\left.+\lambda_{n(+)}\left(1-\theta_{n(+)}+\sum_{j=0}^{\left|z_{k n}\right|} U_{n}(j, j+1)\right)\right]$. Since $\left[\psi_{1}(k), \psi_{2}(k), \ldots, \psi_{n}(k)\right]=\left[\sum_{\ell=1}^{k} y_{\ell 1}, \sum_{\ell=1}^{k} y_{\ell 2}, \ldots, \sum_{\ell=1}^{k} y_{\ell n}\right]$, and $\left\{y_{\ell i}\right\}_{\ell \geq 1, i=1,2, \ldots, n}$, then $\left[U_{1}(j, j+1), U_{2}(j, j+1), \ldots, U_{n}(j, j+1)\right]$ satisfies the renewal theorem as in Feller [29]. Hence, $\left[U_{1}(j, j+1), U_{2}(j, j+1), \ldots, U_{n}(j, j+1)\right]$ is bounded $\forall j$ by a constant. Hence, $\left[M_{1}\left(z_{k 1}\right), M_{2}\left(z_{k 2}\right), \ldots, M_{n}\left(z_{k n}\right)\right] \leq\left[M_{1}(0), M_{2}(0), \ldots, M_{n}(0)\right]+\left[M_{1}, M_{2}, \ldots, M_{n}\right]+\left[M_{1}(\mid\right.$ $\left.\left.z_{k 1} \mid\right), M_{2}\left(\left|z_{k 2}\right|\right), \ldots, M_{n}\left(\left|z_{k n}\right|\right)\right]\left[\tilde{M}_{1}\left(\left|z_{k 1}\right|\right), \tilde{M}_{2}\left(\left|z_{k 2}\right|\right), \ldots, \tilde{M}_{n}\left(\left|z_{k n}\right|\right)\right]$. By the same method, we can show that $\left[L_{1}\left(z_{k 1}\right), L_{2}\left(z_{k 2}\right), \ldots, L_{n}\left(z_{k n}\right)\right] \leq\left[\tilde{L}_{1}\left(\left|z_{k 1}\right|\right), \tilde{L}_{2}\left(\left|z_{k 2}\right|\right), \ldots, \tilde{L}_{n}\left(\left|z_{k n}\right|\right)\right]$ in the left parts of $L_{i}, i=1,2, \ldots, n$. 
In addition, to the conditions in the previous theorems, we need to prove another important condition that is $E\left|Z_{0}\right|<\infty$. This condition confirms the existence of the finiteness.

Theorem 3: If $\left(\phi_{1}, \phi_{2}, \ldots, \phi_{n}, \bar{\phi}_{1}, \bar{\phi}_{2}, \ldots, \bar{\phi}_{n}\right) \in \Phi_{0}$ are a combination of finite search, then $E\left|Z_{0}\right|$ is finite.

Proof It is clear that $E(\tau)<\infty$ if $(P(\tau$ is finite $)=1$. Thus, we have

$$
\begin{aligned}
P(\tau \text { is finite })= & P\left(\tau_{\phi_{1}} \text { is finite or } \tau_{\phi_{2}} \text { is finite or...or } \tau_{\phi_{n}} \text { is finite or } \tau_{\bar{\phi}_{1}} \text { is finite or } \tau_{\bar{\phi}_{2}}\right. \text { is finite } \\
& \text { or ... or } \left.\tau_{\bar{\phi}_{n}} \text { is finite }\right) \\
= & \bigcup_{i=1}^{n}\left(P\left(\tau_{\phi_{i}} \text { is finite }\right)+P\left(\tau_{\bar{\phi}_{i}} \text { is finite }\right)\right) \\
= & \sum_{i=1}^{n}\left(P\left(\tau_{\phi_{i}} \text { is finite }\right)+P\left(\tau_{\bar{\phi}_{i}} \text { is finite }\right)\right) .
\end{aligned}
$$

But, we have only one of $\left(P\left(\tau_{\phi_{i}}\right.\right.$ is finite $)=1$ or $P\left(\tau_{\bar{\phi}_{i}}\right.$ is finite $)=1$ for all $i=1,2, \ldots, n$. If we suppose that $P\left(\tau_{\phi_{i}}\right.$ is finite $)+P\left(\tau_{\bar{\phi}_{i}}\right.$ is finite $)=1$, then we have $Z_{0}=\phi_{i}\left(\tau_{\phi_{i}}\right)-B\left(\tau_{\phi_{i}}\right.$ )$+\bar{\phi}_{i}\left(\tau_{\bar{\phi}_{i}}\right)-B\left(\tau \bar{\phi}_{i}\right)=\phi_{i}\left(\tau_{\phi_{i}}\right)+\bar{\phi}_{i}\left(\tau_{\bar{\phi}_{i}}\right)-\left(B\left(\tau_{\phi_{i}}\right)+B\left(\tau_{\bar{\phi}_{i}}\right)\right)$ with probability one and hence, $\left|Z_{0}\right| \leq\left|\phi_{i}\left(\tau_{\phi_{i}}\right)+\bar{\phi}_{i}\left(\tau_{\bar{\phi}_{i}}\right)\right|+\left|B\left(\tau_{\phi_{i}}\right)+B\left(\tau_{\bar{\phi}_{i}}\right)\right| \leq \tau_{\phi_{i}}+\tau_{\bar{\phi}_{i}}+\mid B\left(\tau_{\phi_{i}}\right)+B\left(\tau_{\bar{\phi}_{i}}\right)$ $|, E| Z_{0}\left|\leq E\left(\tau_{\phi_{i}}\right)+E\left(\tau_{\bar{\phi}_{i}}\right)+E\right| B\left(\tau_{\phi_{i}}\right)|+E| B\left(\tau_{\bar{\phi}_{i}}\right) \mid$. But, $\left|B\left(\tau_{\phi_{i}}\right)\right| \leq \tau_{\phi_{i}}$ and $\mid B\left(\tau_{\bar{\phi}_{i}}\right)$ $\mid \leq \tau_{\bar{\phi}_{i}}$, then $E\left|B\left(\tau_{\phi_{i}}\right)+B\left(\tau_{\bar{\phi}_{i}}\right)\right| \leq E\left(\tau_{\phi_{i}}+\tau_{\bar{\phi}_{i}}\right)$ leads to $E\left|B\left(\tau_{\phi_{i}}\right)\right|+E\left|B\left(\tau_{\bar{\phi}_{i}}\right)\right| \leq E($ $\left.\tau_{\phi_{i}}\right)+E\left(\tau_{\bar{\phi}_{i}}\right)$. If $E\left(\tau_{\phi_{i}}\right)<\infty$ and $E\left(\tau_{\bar{\phi}_{i}}\right)<\infty$, then $E\left|B\left(\tau_{\phi_{i}}\right)\right|<\infty, E\left|B\left(\tau_{\bar{\phi}_{i}}\right)\right|<\infty$, and $E\left|Z_{0}\right|$ is finite. Also, if $P\left(\tau_{\phi_{\hbar}}\right.$ is finite $)+p\left(\tau_{\bar{\phi}_{\hbar}}\right.$ is finite $)=1$ for all $\hbar \neq i, i=1,2, \ldots, n$ then $Z_{0}=\phi_{i}\left(\tau_{\phi_{i}}\right)-B\left(\tau_{\phi_{i}}\right)+\bar{\phi}_{i}\left(\tau_{\bar{\phi}_{i}}\right)-B\left(\tau_{\bar{\phi}_{i}}\right)=\phi_{i}\left(\tau_{\phi_{i}}\right)+\bar{\phi}_{i}\left(\tau_{\bar{\phi}_{i}}\right)-\left(B\left(\tau_{\phi_{i}}\right)+B\left(\tau_{\bar{\phi}_{i}}\right)\right)$ with probability one; similarly, we get $E\left|Z_{0}\right|$ is finite.

The direct result to the realization of the previous theorems confirming a finite search plan if $E\left|Z_{0}\right|$ is finite.

\section{Computational expected value for $\boldsymbol{\tau}$}

From the Markovian property of the Brownian motion and its dependence on normal distribution, we can use this information to compute $E(\tau)$ which depends on the probability of meeting the target on the line $L_{i}, i=1,2, \ldots, n$ at the time step $k$. This probability is given by $G_{(2 k-1) i}^{(+)}$which is depending on the probability of the meeting at the time $G_{(2 k-3) i}^{(+)}$.

Theorem 4: The approximated value of $E(\tau)$ is given by

$$
\begin{gathered}
E(\tau)=\sum_{i=1}^{n}\left(\lambda_{i(+)} \theta_{i(+)}+\lambda_{i(+)} \theta_{i(+)}\left(\theta_{i(+)}^{2}-1\right) \sum_{k=2}^{\infty} \theta_{i(+)}^{2 k-2} \int_{0}^{\infty} \int_{-\infty}^{H_{(2 k-3) i}^{(+)}-z_{i}}\left[1-\Phi\left(G_{(2 k-5) i}^{(+)}-z_{i}\right)\right]\right. \\
d \Phi\left(G_{(2 k-5) i}^{(+)}-z_{i}\right) \gamma_{i}\left(d z_{i}\right)+\lambda_{i(-)} \theta_{i(-)}+\lambda_{i(-)} \theta_{i(-)}^{2}\left(\theta_{i(-)}^{2}-1\right) \\
\left.\sum_{k=2}^{\infty} \theta_{(-)}^{2 k-2} \int_{-\infty}^{0} \int_{-\infty}^{H_{(2 k-2) i}^{(-)}+z_{i}} \Phi\left(G_{(2 k-4) i}^{(-)}+z_{i}\right) d \Phi\left(G_{(2 k-4) i}^{(-)}+z_{i}\right) \gamma_{i}\left(d z_{i}\right)\right) .
\end{gathered}
$$


where $\left(G_{(2 k-5) i}^{(+)}-z_{i}\right) \sim N\left(0,\left(G_{(2 k-3) i}^{(+)}-G_{(2 k-5) i}^{(+)}\right)\right) \quad$ and $\quad\left(G_{(2 k-4) i}^{(-)}+z_{i}\right) \sim N\left(0,\left(G_{(2 k-2) i}^{(-)} i^{-}\right.\right.$ $\left.\left.G_{(2 k-4) i}^{(-)}\right)\right)$with the distribution functions $\Phi\left(G_{(2 k-5) i}^{(+)}-z_{i}\right)$ and $\Phi\left(G_{(2 k-4) i}^{(-)}+z_{i}\right)$, respectively, at time step $\mathrm{k}$ on the line $\mathrm{L}_{i}, i=1,2, \ldots, n$.

Proof From (5), we get

$$
\begin{aligned}
E(\tau)= & \sum_{i=1}^{n}\left(\lambda_{i(+)} \theta_{i(+)} P\left(\tau_{\phi_{i}}>0\right)+\lambda_{i(+)} \theta_{i(+)}\left(\theta_{i(+)}^{2}-1\right)\right. \\
& \sum_{k=2}^{\infty} \theta_{i(+)}^{2 k-2} P\left(\tau_{\phi_{i}}>G_{(2 k-3) i}^{(+)}\right)+\lambda_{i(-)} \theta_{i(-)} P\left(\tau_{\bar{\phi}_{i}}>0\right) \\
& \left.+\lambda_{i(-)} \theta_{i(-)}^{2}\left(\theta_{i(-)}^{2}-1\right) \sum_{k=2}^{\infty} \theta_{(-)}^{2 k-2} P\left(\tau_{\bar{\phi}_{i}}>G_{(2 k-2) i}^{(-)}\right)\right) .
\end{aligned}
$$

Also, at the time step $k$ on the right part of the line $L_{i}, i=1,2, \ldots, n$, we have

$$
P\left(\tau_{\phi_{i}}>\left(G_{(2 k-3) i}^{(+)}\right) \leq \int_{0}^{\infty} P\left(Z_{0 i}+B\left(G_{(2 k-3) i}^{(+)}\right)>\left(H_{(2 k-3) i}^{(+)}\right) \mid Z_{0 i}=z_{i}\right) \gamma_{i}\left(d z_{i}\right) .\right.
$$

Consequently, we can find that

$$
\begin{aligned}
& P\left(Z_{0 i}+B\left(G_{(2 k-3) i}^{(+)}\right)>H_{(2 k-3) i}^{(+)} \mid Z_{0 i}=z_{i}\right)=P\left(\left(B\left(G_{(2 k-3) i}^{(+)}\right)>\left(H_{(2 k-3) i}^{(+)}-z_{i}\right)\right.\right. \\
& \quad=1-P\left(B\left(G_{(2 k-3) i}^{(+)} \leq\left(H_{(2 k-3) i}^{(+)}-z_{i}\right)\right) .\right.
\end{aligned}
$$

Generally, for any times $t, s$, we have $B(t)-B(s)$ is a normal random variable with mean 0 and variance $t-s$ where $s<t$. Therefore, we can find that

$$
\begin{aligned}
& P\left(B\left(G_{(2 k-3) i}^{(+)}\right) \leq\left(H_{(2 k-3) i}^{(+)}-z_{i}\right)\right) \leq \int_{-\infty}^{\infty} P\left(B\left(G_{(2 k-5) i}^{(+)}\right) \leq\left(H_{(2 k-5) i}^{(+)}-z_{i}\right),\left[B\left(G_{(2 k-3) i}^{(+)}\right)\right.\right. \\
& \left.\left.+B\left(G_{(2 k-5) i}^{(+)}\right)-B\left(G_{(2 k-5) i}^{(+)}\right)\right] \leq\left(H_{(2 k-5) i}^{(+)}-z_{i}\right) \mid B\left(G_{(2 k-5) i}^{(+)}\right)=\left(G_{(2 k-5) i}^{(+)}-z_{i}\right)\right) \\
& \times \frac{1}{\left(G_{(2 k-3) i}^{(+)}-G_{(2 k-5) i}^{(+)}\right) \sqrt{2 \pi}} \times e^{-\frac{1}{2}\left(\frac{G_{(2 k-5) i^{-2}}^{(+)}}{\left(G_{(2 k-3) i}^{-G_{(2 k-5) i}}\right.}\right)^{2}} d\left(G_{(2 k-5) i}^{(+)}-z_{i}\right) \\
& =\int_{-\infty}^{\infty} P\left(\left[B\left(G_{(2 k-3) i}^{(+)}\right)+B\left(G_{(2 k-5) i}^{(+)}\right)\right]-B\left(G_{(2 k-5) i}^{(+)}\right) \leq\left(H_{(2 k-5) i}^{(+)}-z_{i}\right) \mid B\left(G_{(2 k-5) i}^{(+)}\right)=\left(G_{(2 k-5) i}^{(+)}-z_{i}\right)\right) \\
& \times \frac{1}{\left(G_{(2 k-3) i}^{(+)}-G_{(2 k-5) i}^{(+)}\right) \sqrt{2 \pi}} e^{-\frac{1}{2}\left(\frac{G_{(2 k-5) i}^{\left(+-z_{i}\right.}}{\left(G_{(2 k-3) i}^{(+)} G_{(2 k-5) i}^{(+)}\right)}\right)^{2}} d\left(G_{(2 k-5) i}^{(+)}-z_{i}\right)
\end{aligned}
$$

(see Klebaner [30]). By considering that the first meeting is not done at the time $t$ $\left.=G_{(2 k-5) i}^{(+)}\right)$, then 


$$
\begin{aligned}
& P\left(B\left(G_{(2 k-3) i}^{(+)} \leq\left(H_{(2 k-3) i}^{(+)}-z_{i}\right)\right)=\int_{-\infty}^{H_{(2 k-3) i}^{(+)} z_{i}} P\left(\left[\left(B\left(G_{(2 k-3) i}^{(+)}\right) \leq\left(H_{(2 k-3) i}^{(+)}-z_{i}\right)\right]-\left[\left(B\left(G_{(2 k-5) i}^{(+)}\right) \leq\left(H_{(2 k-5) i}^{(+)}\right)-z_{i}\right)\right]\right.\right.\right. \\
& \left.\leq-\left(G_{(2 k-5) i}^{(+)}-z_{i}\right)\right) \times \frac{1}{\left(G_{(2 k-3) i}^{(+)}-G_{(2 k-5) i}^{(+)}\right) \sqrt{2 \pi}}
\end{aligned}
$$

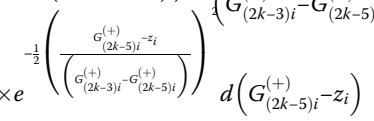

$$
\begin{aligned}
& =\int_{-\infty}^{H_{(2 k-3) i}^{(+)}-z_{i}} \Phi\left(-\left(G_{(2 k-5) i}^{(+)}-z_{i}\right) d \Phi\left(G_{(2 k-5) i}^{(+)}-z_{i}\right)=\int_{-\infty}^{H_{2 i-3}^{(+)}-z}\left[1-\Phi\left(G_{(2 k-5) i}^{(+)}-z_{i}\right)\right] d \Phi\left(G_{(2 k-5) i}^{(+)}-z_{i}\right),\right.
\end{aligned}
$$

where $\left(G_{(2 k-5) i}^{(+)}-z_{i}\right) \sim N\left(0,\left(G_{(2 k-3) i}^{(+)}-G_{(2 k-5) i}^{(+)}\right)\right)$and $\Phi\left(G_{(2 k-5) i}^{(+)}-z_{i}\right)$ is the distribution function of this variable. By a similar manner, on the left part of line $L_{i}, i=1,2, \ldots, n$, one can compute the probability at time step $k$ by

$$
\begin{gathered}
P\left(Z_{0 i}+B\left(G_{(2 k-2) i}^{(-)}\right)<H_{(2 k-2) i}^{(-)} \mid Z_{0 i}=z_{i}\right)=P\left(B\left(G_{(2 k-2) i}^{(-)}\right)-\left(H_{(2 k-2) i}^{(-)}+z_{i}\right)<0\right) \\
=\int_{-\infty}^{H_{(2 k-2) i}^{(-)}+z_{i}} \Phi\left(G_{(2 k-2) i}^{(-)}+z_{i}\right) d \Phi\left(G_{(2 k-2) i}^{(-)}+z_{i}\right) .
\end{gathered}
$$

Since the target starts its motion on the line $L_{i}, i=1,2, \ldots, n$ from a random point $z_{i}$, then we can assume that $P\left(\tau_{\phi_{i}}>0\right)=P\left(\tau_{\bar{\phi}_{i}}>0\right)=1$. In addition, the first interviewing time should be done on one real line by one searcher. Consequently, in (10), we can obtain the approximate value of $E(\tau)$ by

$$
\begin{aligned}
E(\tau) & =\sum_{i=1}^{n}\left(\lambda_{i(+)} \theta_{i(+)}+\lambda_{i(+)} \theta_{i(+)}\left(\theta_{i(+)}^{2}-1\right) \sum_{k=2}^{\infty} \theta_{i(+)}^{2 k-2} \int_{0}^{\infty} \int_{-\infty}^{H_{(2 k-3) i}^{(+)} z_{i}}\right. \\
& {\left[1-\Phi\left(G_{(2 k-5) i}^{(+)}-z_{i}\right)\right] d \Phi\left(G_{(2 k-5) i}^{(+)}-z_{i}\right) \gamma_{i}\left(d z_{i}\right)+\lambda_{i(-)} \theta_{i(-)} } \\
& \left.+\lambda_{i(-)} \theta_{i(-)}^{2}\left(\theta_{i(-)}^{2}-1\right) \sum_{k=2}^{\infty} \theta_{(-)}^{2 k-2} \int_{-\infty}^{0} \int_{-\infty}^{H_{(2 k-2) i}^{(-)}+z_{i}} \Phi\left(G_{(2 k-4) i}^{(-)}+z_{i}\right) d \Phi\left(G_{(2 k-4) i}^{(-)}+z_{i}\right) \gamma_{i}\left(d z_{i}\right)\right) .
\end{aligned}
$$

If we have $X \sim N\left(0, \sigma^{2}\right)$, then $\int_{-\infty}^{0} d \Phi(x)=\frac{1}{2}$. This leads to $\frac{1}{2}<\int_{-\infty}^{H_{2 i-3}^{(+)}-z} d \Phi\left(G_{2 i-5}^{(+)}-z\right)<1$ where $H_{(2 k-3) i}^{(+)}-z_{i} \quad H_{(2 k-3) i}^{(+)}-z_{i} \quad H_{(2 k-3) i}^{(+)}-z_{i}$ $\int_{-\infty}^{(2 k-3) i}\left[1-\Phi\left(G_{(2 k-5) i}^{(+)}-z_{i}\right)\right] d \Phi\left(G_{2 i-5}^{(+)}-z_{i}\right)=\int_{-\infty}^{(2 k-3) i} d \Phi\left(G_{(2 k-5) i}^{(+)}-z_{i}\right)-\int_{-\infty}^{(2 k-3) i} \Phi\left(G_{(2 k-5) i}^{(+)}-z_{i}\right) d \Phi\left(G_{(2 k-5) i}^{(+)}\right.$ $\left.z_{i}\right)$ and $H_{(2 k-3) i}^{(+)}-z_{i}>0$. From the fact $\int_{-\infty}^{0} \Phi(x) d \Phi(x)=\int_{0}^{\frac{1}{2}} x d x=\frac{1}{8}<\frac{1}{2}$, one can easily con-

$$
H_{(2 k-3) i}^{(+)}-z_{i}
$$

clude that $0<\int_{-\infty}^{(2 k-3) i} \Phi\left(G_{(2 k-5) i}^{(+)}-z_{i}\right) d \Phi\left(G_{(2 k-5) i}^{(+)}-z_{i}\right)<\frac{1}{2}$. This also contributes to the assertiveness of the existence and finiteness of our search plan where the Brownian target is not evading the searchers.

\section{Concluding remarks}

1) The existence of a cooperative search plan with multiple searchers that used the coordinated linear search technique to find a Brownian target on one of $n$ real line has been presented. 
2) The random variable $Z_{0}$ is used to give the target initial position.

3) We showed the finiteness of our continuous search plan by giving the conditions that make $E(\tau)$ is finite as in Theorem 1.

4) At any time step $k$, we showed that the chosen search plan $\Phi_{0}=\left\{(\Phi, \bar{\Phi}): \phi_{i} \in \Phi\right.$, $\left.\bar{\phi}_{i} \in \bar{\Phi} \forall i=1,2, \ldots, n\right\}$ should satisfy $\left[M_{1}\left(z_{k 1}\right), M_{2}\left(z_{k 2}\right), \ldots, M_{n}\left(z_{k n}\right)\right] \leq\left[\tilde{M}_{1}\left(\left|z_{k 1}\right|\right), \tilde{M}_{2}\right.$ $\left.\left(\left|z_{k 2}\right|\right), \ldots, \tilde{M}_{n}\left(\left|z_{k n}\right|\right)\right]$, and $\left[L_{1}\left(z_{k 1}\right), L_{2}\left(z_{k 2}\right), \ldots, L_{n}\left(z_{k n}\right)\right] \leq\left[\tilde{L}_{1}\left(\left|z_{k 1}\right|\right), \tilde{L}_{2}\left(\left|z_{k 2}\right|\right), \ldots, \tilde{L}_{n}(\mid\right.$ $\left.\left.z_{k n} \mid\right)\right]$ where $\left[\tilde{M}_{1}\left(\left|z_{k 1}\right|\right), \tilde{M}_{2}\left(\left|z_{k 2}\right|\right), \ldots, \tilde{M}_{n}\left(\left|z_{k n}\right|\right)\right]$ and $\left[\tilde{L}_{1}\left(\left|z_{k 1}\right|\right), \tilde{L}_{2}\left(\left|z_{k 2}\right|\right), \ldots, \tilde{L}_{n}\left(\left|z_{k n}\right|\right)\right.$ ] are vectors of linear functions, see Theorem 2 .

5) Besides proving that $E\left|Z_{0}\right|<\infty$ in Theorem 3, we compute the approximated value of $E(\tau)$ in Theorem 4 .

6) In future research, one can study the finiteness of the expected value of the first meeting time between one of $n$-cooperative searchers and $n$-dimensional Brownian target and calculate this expected value.

\section{Acknowledgements}

The author gratefully acknowledges the anonymous referees for their insightful and constructive comments and suggestions.

Author's contributions

The author read and approved the final manuscript.

\section{Funding}

Not applicable

\section{Availability of data and materials}

Not applicable

\section{Competing interests}

The author declares that he has no competing interests.

Received: 21 February 2019 Accepted: 5 November 2019

Published online: 09 January 2020

\section{References}

1. Mohamed, A., Kassem, M., El-Hadidy, M.: Multiplicative linear search for a Brownian target motion. Applied Mathematical Modelling. 35(9), 4127-4139 (2011) https://doi.org/10.1016/j.apm.2011.03.024

2. El-Hadidy, M.: Searching for a d-dimensional Brownian target with multiple sensors. International Journal of Mathematics in Operational Research. 9(3), 279-301 (2016) https://doi.org/10.1504/IJMOR.2016.078822

3. El-Rayes, A., Mohamed, A., Abou, G.H.: Linear search for a Brownian target motion. Acta Mathematica Scientia. 23(B3), 321-327 (2003) https://doi.org/10.1016/S0252-9602(17)30338-7

4. Corwin, T.: On Search for a Brownian Target. Journal of Applied Probability. 17, 243-247 (1980)

5. Reyniers, D.: Coordinated Search for an Object on the Line. European Journal of Operational Research. 95(3), 663-670 (1996) https://doi.org/10.1016/S0377-2217(96)00314-1

6. Reyniers, D.: Coordinated Two Searchers for an Object Hidden on an Interval. Journal the Operational Research Society. 46(11), 1386-1392 (1995)

7. El-Hadidy, M., Abou-Gabal, H.: Coordinated Search for a Random Walk Target Motion. Fluctuation and Noise Letters. 17(1), 1850002 (2018) (11 pages). https://doi.org/10.1142/S0219477518500025

8. Mohamed, A., Abou-Gabal, H., El-Hadidy, M.: Coordinated search for a randomly located target on the plane. European Journal of Pure and Applied Mathematics. 2(1), 97-111 (2009)

9. Mohamed, A., Fergany, H., El-Hadidy, M.: On the coordinated search problem on the plane. Istanbul Business Research. 41(1), 80-102 (2012)

10. El-Hadidy, M., Teamah, A., El-Bagoury, A.: 3-Dimensional coordinated search technique for a randomly located target. International Journal of Computing Science and Mathematics. 9(3), 258-272 (2018) https://doi.org/10.1504/IJCSM.2018. 093152

11. Kassem, M., El-Hadidy, M.: Optimal multiplicative Bayesian search for a lost target. Applied Mathematics and Computation. 247, 795-802 (2014) https://doi.org/10.1016/j.amc.2014.09.039

12. El-Hadidy, M.: Optimal spiral search plan for a randomly located target in the plane. International Journal of Operational Research. 22(4), 454-465 (2015) https://doi.org/10.1504/IJOR.2015.068561

13. El-Hadidy, M.: Optimal searching for a helix target motion. Science China Mathematics. 58(4), 749-762 (2015) https://doi. org/10.1007/s11425-014-4864-5

14. Mohamed, A., Kassem, M., El-Hadidy, M.: M-States search problem for a lost target with multiple sensors. International Journal of Mathematics in Operational Research. 10(1), 104-135 (2017) https://doi.org/10.1504/IJMOR.2017.080747

15. Mohamed, A., Abou-Gabal, H., El-Hadidy, M.: Random Search in a bounded area. International Journal of Mathematics in Operational Research. 10(2), 137-149 (2017) https://doi.org/10.1504/IJMOR.2017.081921 
16. El-Hadidy, M.: Fuzzy Optimal search plan for N-dimensional randomly moving target. International Journal of Computational Methods. 13(6), 1650038 (2016) (38 pages). https://doi.org/10.1142/S0219876216500389

17. El-Hadidy, M.: On maximum discounted effort reward search problem. Asia-Pacific Journal of Operational Research. 33(3), 1650019 (2016) (30 pages). https://doi.org/10.1142/50217595916500196

18. El-Hadidy M. On the existence of a finite linear search plan with random distances and velocities for a one-dimensional brownian target. International Journal of Operational Research. (2018). In Press. http://www.inderscience.com/info/ ingeneral/forthcoming.php?jcode=ijor

19. El-Hadidy, M.: Existence of finite parabolic spiral search plan for a Brownian Target. International Journal of Operational Research. 31(3), 368-383 (2018) https://doi.org/10.1504/IJOR.2018.089737

20. El-Hadidy M. Generalised linear search plan for a D-dimensional random walk target. International Journal of Mathematics in Operational Research. 15(2), 211-241 (2019) https://doi.org/10.1504/IJMOR.2019.101622.

21. Kagan, E., Ben-Gal, I.: Search and foraging: individual motion and swarm dynamics. CRC Press. (2015)

22. Guerrier, C., Holcman, D.: Brownian search for targets hidden in cusp-like pockets: progress and applications. The European Physical Journal Special Topics. 223(14), 3273-3285 (2014) https://doi.org/10.1140/epjst/e2014-02332-6

23. Palyulin, V., Chechkin, A., Metzler, R.: Space-fractional Fokker-Planck equation and optimization of random search processes in the presence of an external bias. Journal of Statistical Mechanics: Theory and Experiment. 11, P11031 (2014) https://doi.org/10.1088/1742-5468/2014/11/P11031

24. Radmard, S., Croft, E.: Active target search for high dimensional robotic systems. Autonomous Robots. 41(1), 163-180 (2017) https://doi.org/10.1007/s10514-015-9539-8

25. Stone, L., Royset, J., Washburn, A.: Optimal search for moving targets. Part of the International Series in Operations Research \& Management Science book series. Springer, Cham (2016). https://doi.org/10.1007/978-3-319-26899-6

26. Jia, Q., Xu, H., Feng, X., Gu, H., Gao, L.: Research on cooperative area search of multiple underwater robots based on the prediction of initial target information. Ocean Engineering. 172, 660-670 (2019)

27. El-Hadidy, M., Alzulaibani, A.: Cooperative search model for finding a Brownian target on the real line. Journal of Taibah University for Science. 13(1), 177-183 (2019) https://doi.org/10.1080/16583655.2018.1552493.

28. El-Hadidy M, Alfreedi A, Alzulaibani A. Optimal multiplicative generalized coordinated search technique to find a Ddimensional random walker. International Journal of Operational Research, (2019), Accepted for Publication. https://doi. org/10.1504/IJOR.2021.10024709.

29. Feller, W.: An Introduction to Probability Theory and Its Applications, vol. II. Wiley, New York (1966)

30. Klebaner, F.: Introduction to Stochastic Calculus with Applications. Imperial College Press. (1998)

\section{Publisher's Note}

Springer Nature remains neutral with regard to jurisdictional claims in published maps and institutional affiliations.

\section{Submit your manuscript to a SpringerOpen ${ }^{\circ}$ journal and benefit from:}

- Convenient online submission

- Rigorous peer review

- Open access: articles freely available online

High visibility within the field

- Retaining the copyright to your article

Submit your next manuscript at $\boldsymbol{\nabla}$ springeropen.com 